\title{
TOWARDS SONAR BASED PERCEPTION AND MODELLING FOR UNMANNED UNTETHERED UNDERWATER VEHICLES
}

\author{
B. Steer, J. Kloske, P. Garner, L. LeBlanc and S. Schock.* \\ (407) 367-2810, EMail: steer@blueplanet.oe.fau.edu
}

February 12, 1993

\begin{abstract}
The ability to automatically generate internal models from sensed data will be of critical importance in the development of flexible, adaptive and useful unmanned underwater vehicles. The subsequent recognition of objects based on these a-priori models, which may be incomplete or partial, will also be an essential component in the success of UUV missions. This paper describes and presents experimental results obtained with our calibrated computer controlled frequency modulated (FM) subbottom penetrating sonar. We present a topographic model of the sea-floor surface and a sequence of sonar "images", obtained with the same FM sonar. This latter sequence shows a vertical section through the sea-bed itself. Despite the absence of surface features the variations in stratigraphy of this vertical section and the automation of the interpretation of these features opens the possibiliity of using these data to navigate when resurfacing is either not an option or desirable, for example, during under ice missions or from full ocean depth.
\end{abstract}

\section{INTRODUCTION}

The ability to automatically generate internal models from sensed data will be of critical importance in the development of flexible, adaptive and useful unmanned underwater vehicles. The subsequent recognition of objects based on these a-priori models, which may be incomplete or partial, will also be an essential component in the success of UUV missions. The spatial extent over which these sensor derived models may need to cover will vary as will the resolution at which individual "features" need to be modelled. For example, horizontal scales ranging from $100 \mathrm{~km}^{2}$ to $10 \mathrm{~m}^{2}$ with corresponding resolutions of order \pm 10 to \pm 0.01 metres are quoted as real requirements. If accurate sensor based models

*Advanced Marine Systems Group, Department of Ocean Engineering, College of Engineering, Florida Atlantic University, Boca Raton, FL 33431 USA or maps can be made automatically this also open the possibility of UUV's using terrain aided comparison techniques to navigate. This would be attractive possibility in circumstances when resurfacing to obtain GPS fixes is not possible or desirable, for example, during under-ice missions, or from full ocean depth.

Separately, the development of automated and highly sensitive scientific and commercial acoustic tools are needed in diverse fields including sea-floor seismics, physical and geological oceanography, environmental and geotechnical engineering, hydrology and marine archeology. The development of new data collection techniques and imaging techniques will yield significant cost savings over existing methods. When developed to work on UUVs they will allow unprecedented insight into the current formation and past processes at work.

Improved and intelligent strategies to control the attention and exploratory activities of unmanned underwater robot-systems are also required since a key element of intelligent behaviour is the ability to quickly and correctly assess a situation and to act or react accordingly [2]. As unmanned untethered underwater robot-systems are applied in environments that are less constrained and more unstructured, they will have to make intelligent, online, time-constrained choices as to what is important to observe and how to acquire observations in order to supply sufficient information for effective reasoning and action [2].

To place this component of our research into context we exhibit figure 1 . This shows the generic functional blocks for a total underwater robot-system as envisaged by Russell and Dunbar [5]. There is an approximate division into sensing and control. This paper presents results which will support other processes that will enable a UUV to better reason about the tasks it has to perform, detect changes in the task domain, locate objects of interest and navigate ${ }^{1}$ through uncertain envi-

\footnotetext{
${ }^{1}$ Navigation is taken to mean a process which establishes ("fixes") the position and attitude of the robot-system rela-
} 


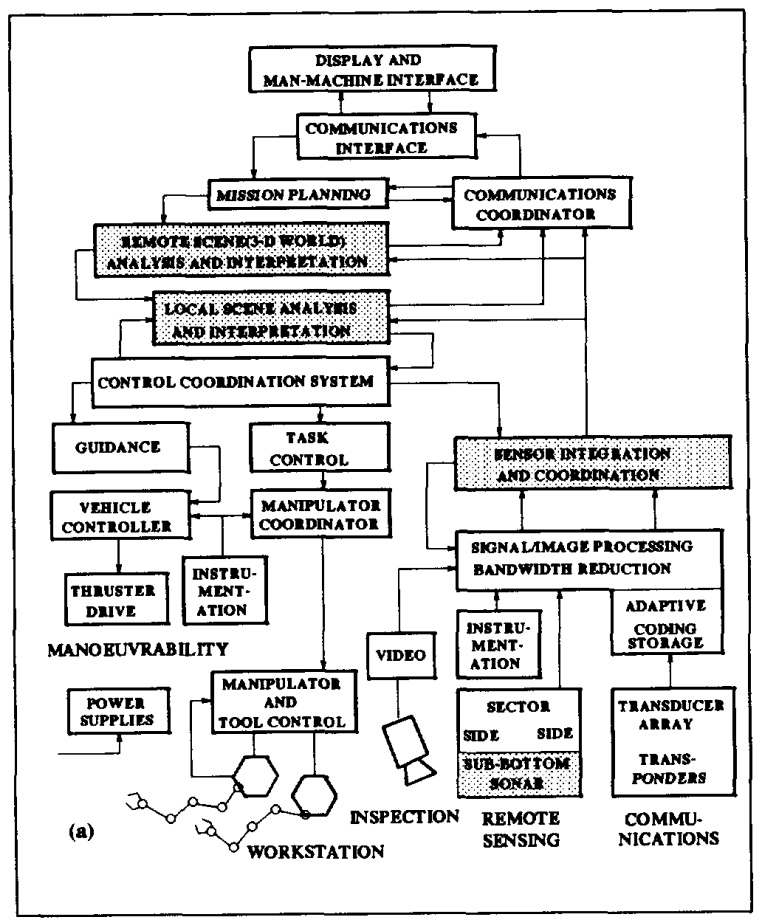

Figure 1: Total system functional diagram. (After Russell and Dunbar). See text for further details.

ronments.

\section{DEVELOPMENT OF THE FAU-AUV}

The FAU-Perry AUV, the "Ocean Voyager", is a robot submarine project started jointly by Dunn and Perry Technologies ${ }^{2}$ in 1989. It is well understood that building a real robot-vehicle forces those involved to solve more of the real problems than if only simulations are developed. Dunn and Perry Technologies chose to develop a torpedo shaped vehicle. A highly schematic version is shown in figure 2 . Its overall length is $21 \mathrm{ft}(6.4 \mathrm{~m})$ and the outside diameter is 21 in $(0.53 \mathrm{~m})$. Its dry weight is $1800 \mathrm{lbs}$ (about $800-\mathrm{kg}$ ). Its flank speed is 6 knots. The controllable speed range for the submersible is $1.69 \leq \| \mathrm{V}$ $\| \leq 10.13 \mathrm{ft} / \mathrm{sec},(0.51 \leq\|\mathrm{V}\| \leq 3.08 \mathrm{~m} / \mathrm{sec})$. While the duration a submarine can cruise for is related to many factors, with the initially planned lead acid batteries, the cruise time is expected to be about 60 minutes. The CPU running time is estimated to be about 120-180 minutes.

tive to some frame of reference. The frame of reference may be its own positional history or the position of objects in its environment observed by the robot-system.

${ }^{2}$ Perry Technologies: A Martin Marietta Company, Riviera Beach, FL. USA

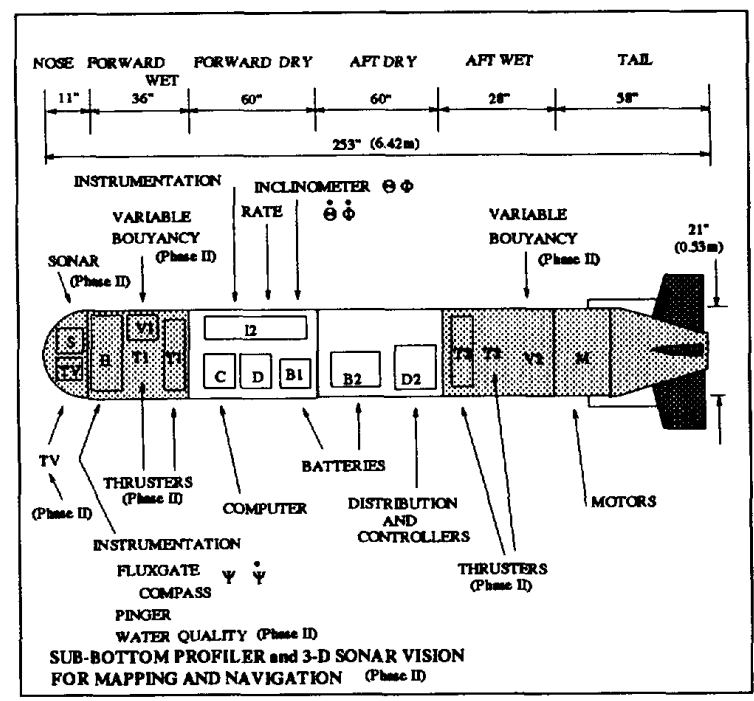

Figure 2: Schematic of FAU Ocean Voyager being developed by Dunn.

These times will increase as higher density energy systems become available. Hydrodynamic design will also improve these figures. Although for this vehicle this was not considered as a design option. Rather the emphasis was intended to be on developing experience throughout the whole design cycle, and in particular the intelligent vehicle control software. The CPU is a 68020 running at $25 \mathrm{MHz}$. The organisation of the sub-sea computers for this vehicle is shown in figure 3 . VxWorks is being used for code developement. This gives us compatability with a number of other institutions. The onboard memory is 1 MByte in size. This clearly needs to increase in size. The power of the vehicle thruster is $3 \mathrm{hp}$. Initially this was designed to be $1 \mathrm{hp}$ but with uncertainty arising from the RF tether induced drag, its size was increased. For attitude control, two $24 \mathrm{v} 8 \mathrm{~A}$ actuators will be used. To provide stabilization information, pitch and roll will be measured with clinometers. The rate of change of pitch roll and yaw will be measured with Watson rate sensors. Heading will be measured with a KVH ROV1000. Altitude will be measured using a Mesotech 807 or 809. Depth will be measured using a Precise Sensors Inc. System sensor. The navigation system is a GPS system onboard a surface vessel with an 9600 baud RF link to a tow float attached to the vehicle. A variety of other sensors are planned to monitor the power and other critical systems. There is space available for a separate bouyancy control system and thruster for and aft. The dry payload is approximately $0.36 \times 0.34 \times 0.3 \mathrm{~m}^{3}$. The vehicle is modular and an additional cylindrical sections 


\section{CHIRP SONAR}

The sonar we are using in our perception and modelling research is computer controlled subbottom profiler that generates high quality seabed reflection data that can be used for imaging subsurface structures and classifying ocean sediments. Using FM pulses that sweep from 1 to $10 \mathrm{kHz}$ the sonar is capable of a vertical resolution of $15 \mathrm{~cm}$ and a subbottom penetration of 10 metres in sand and 100 to 300 metres in silts and clay seabeds. The acoustic pulses can be varied in length from 10 to $200 \mathrm{msec}$. Longer pulses provide the high time bandwidth products needed to improve the signal to noise ratio in the acoustic data during correlation processing. Correlation processing is not only used to improve SNR of the acoustic signal but also is used to compress the long FM signals in time to achieve high vertical resolution. As shown in figure 6 a 80386 based micro-computer with data acquisition and signal processing capability is used to generate an excitation signal for the transmission amplifier and processing subbottom reflection data, displaying subbottom images on a colour monitor and transmitting image data to a hard copy recorder. The $2 \mathrm{~kW}$ transmission amplifier drives 4 piston transducers mounted, for current experimental purposes, in a "towed" fish. This vehicle is typically towed within 10 metres of the sea-floor. Subbottom acoustic reflections are converted to an electrical signal at a line hydraphone array mounted in the aft end of the fish. The projector array provides an effective beam width of 20 degrees during 1 to $10 \mathrm{kHz}$ operation. The result is that it provides quantitative, $(\approx 10 \mathrm{~cm}$ high resolution, deep penetration (this varies from $\approx 10 \mathrm{~m}$ (sand) to $100 \mathrm{~m}$ (clay)) low noise subbottom data. In addition, it generates an acoustic pulse with special frequency domain weighting that provides a nearly constant resolution with depth. A block diagram showing the essential elements of the system are shown in figure 5 .

\section{OUR APPROACH}

Our intention is to exploit the high information content in sequences of chirp subbottom sonar images. There are significant informational advantages in viewing the same scene but from different positions. By adopting technique from the field of machine vision we open the possibility of being able to produce computational descriptions of the source topologies and surface configurations that generated the original image sequence. By judicious use of the information available in the specular reflections and the history of the motion of the vehicle, we can classify the specular echo sources and infer the local structure of the objects bearing them. The motivation for using this information is that the properties of

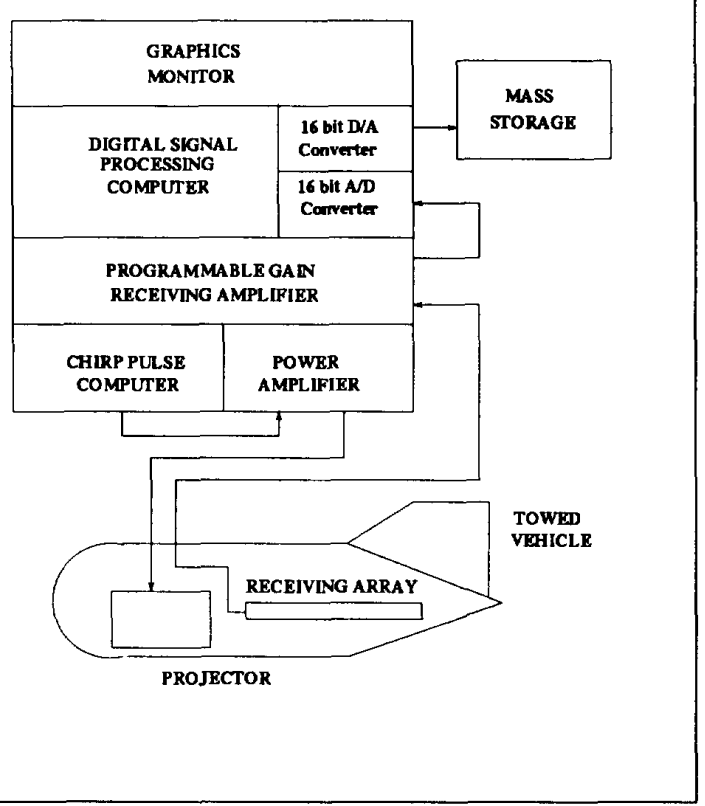

Figure 5: Block diagram of chirp sonar, (after Leblanc and Schock).

the sources are important cues for the map making process. For example, are these observations the result of echoes from a cylinder a sphere or what? Thus this information can be used to drive the object modelling activity and, almost certainly, will need to interact with other processes, for example, processes that annotate maps. Knowledge of the source properties is useful in two main ways, (1) the different source types reflect observer motion in different ways depending on the source topology, and this may be used to construct a correction term for the motion resolution system to account for the source behaviour, and (2) a partial knowledge of the properties of a given source may be used to suggest suitable observer motion strategies for elucidating the information necessary to complete the description of the source.

\section{PRELIMINARY RESULTS}

The data shown in figure 6 was obtained by using the chirp sonar system [7]. The figure shows the results from a geodetic survey off of the Florida coast near FAU in Boca Raton carried out by Kloske [6]. The location of each data point was supplied from a Magnavox MX-200 GPS unit housed onboard our departmental vessel. The depths were obtained from the first return. The dis- 


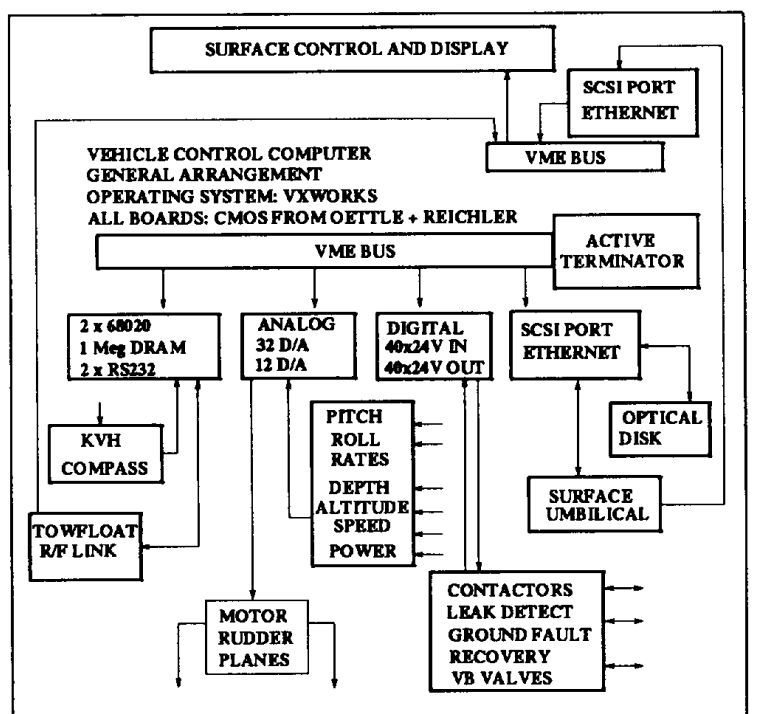

Figure 3: Actual organisation and components of subsea computer systems.

can be added which could house the chirp sonar.

\section{HARDWARE IN THE LOOP SIMULATION}

Shein and Kloske have developed a simulation based on a non-linear model of a submarines six degrees of freedom (6-DOF). The mathematical formulation is based on the David Taylor Naval Ship Research and Development Center report [1]. This simulation allows initial testing and debugging of the AUV software to take place in the laboratory rather than at sea. The simulation runs on a Silicon Graphics system. The simulation is being used to test linear, neural and fuzzy control algorithms. These are described elsewhere [6]. It will allow us to develop and to test navigation and obstacle avoidance algorithms and to generally develop, test and debug the pre-flight and diagnostic software that will be necessary for successful at-sea operations.

\section{SONAR PERCEPTION AND MODELLING}

As Hallam pointed out [3] a new paradigm for sensory interpretation and navigation is required in the marine environment. Algorithms for navigating with land based mobile robot vehicles can assume, quite reasonably, that the cause of sensor motion is self generated. Underwater this assumption is no longer true. Random and systematic forces impose themselves on submersibles disturbing their position and velocity from that expected exacerbating the correspondence problem. Hallam [3] informally defines a sonar interpreter in terms of the task it performs.

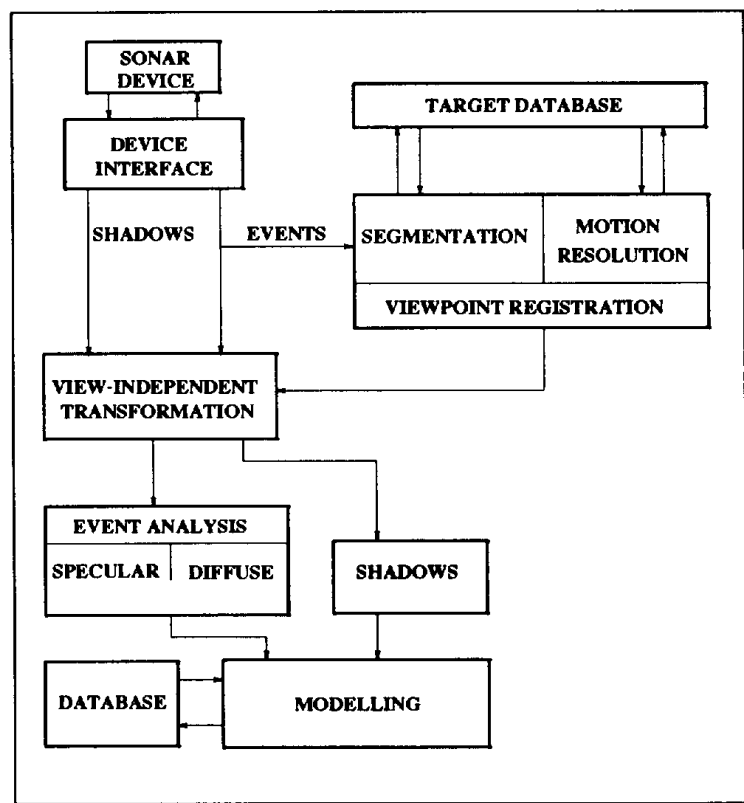

Figure 4: Organisation of a sonar interpreter for underwater robots, (After Hallam). See text for further details.

\begin{abstract}
A sonar intepreter has the task of using acoustic data gathered by a physical sonar attached to an underwater freely mobile "platform" to construct detailed extero-centric three-dimensional computational models of the shape of the seabed and objects in the vehicle's environment. Its task is also to deduce the positions and velocities of those objects and the observer with respect to a fixed viewpoint independent frame of reference.
\end{abstract}

A sonar intepreter should take into account the following: (1) The description it produces should be view independent, (2) the system must be real-time ${ }^{3},(3)$ objects will be visible irregularly and at random, (4) information concerning both diffuse and specular reflections should be used, (5) much of the time an object will be invisible (in this situation range shadows contain useful information), and, (6) as the information content of the input falls the quality of the information from the sonar interpreter ought to degrade gracefully. It is also desirable to make the information control mechanisms flow bidirectionally. An outline of the key elements a sonar interpreter is shown in figure 4 .

\footnotetext{
${ }^{3}$ By real-time we mean producing output instructions at a rate sufficient for the task
} 


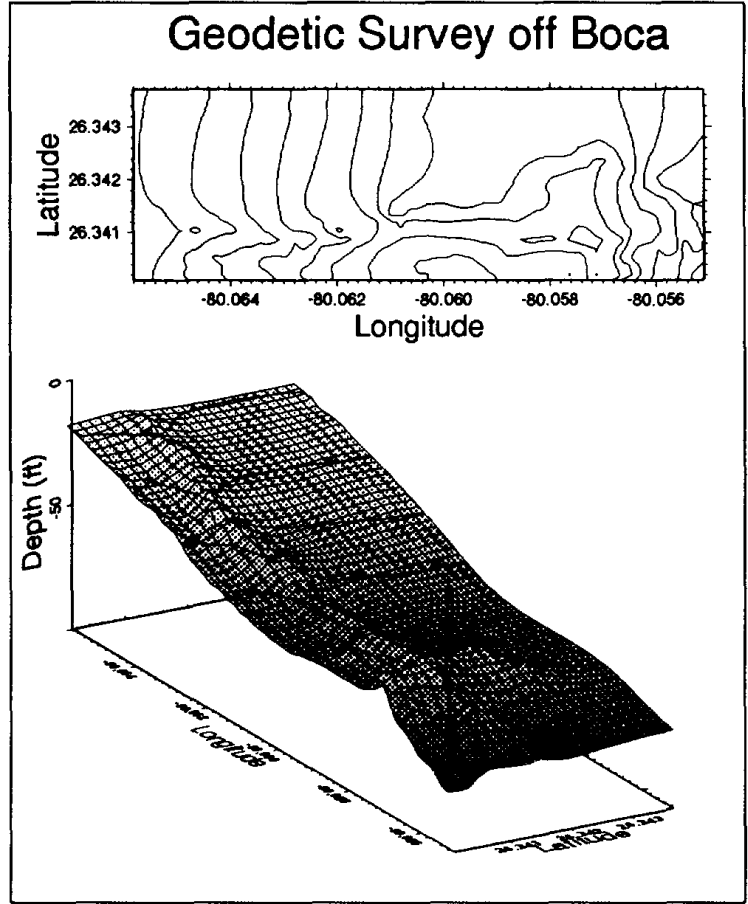

Figure 6: Figure shows preliminary results obtained with chirp sonar of surface features. See text for further details.

tance between samples is of order 60 feet. This figure only shows the first return that is the sea-floor. Figure 7 exhibits data that shows detail of a vertical section through the sea-floor. As we place subsequent transects side by side we generate 3-dimensional "solid" images.

\section{CONCLUSIONS AND FUTURE WORK}

Equipped with these data gathered at-sea, and a full operational capability to obtain more, our current and future work is aimed at extending our modelling capability from these sensed data. This will involve developing the capability to extract features, match them from location to location, and to reconstruct real underwater scenes. In this way more autonomy will be developed in unmanned untethered underwater vehicles.

\section{References}

[1] Feldman. J. David Taylor Naval Ship Research and Development Center revised standard submarine equations of motion. Ship Performance Department. June 1979.

[2] Hager, G. D., Task directed sensor fusion and planning: A computational approach. Kluwer Academic Publishers. Norwell MA. 1990.

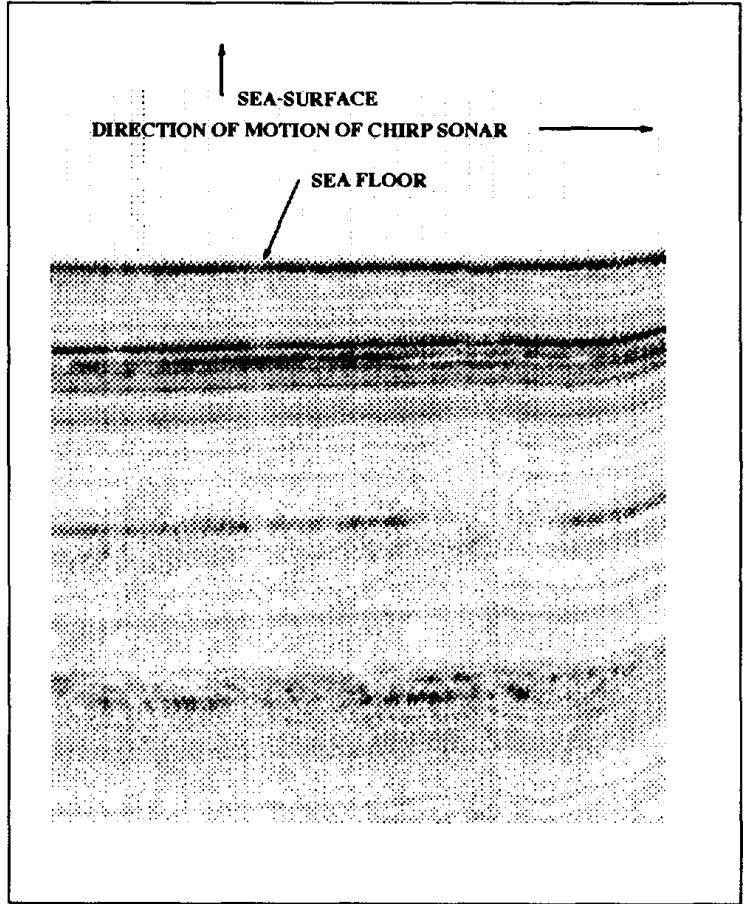

Figure 7: Side view of vertical section of ocean floor obtained using chirp sonar. Vertical axis is depth into ocean floor. See text for further details.

[3] Hallam, J. Intelligent automatic interpretation of active marine sonar. Ph.D Thesis, University of Edinburgh. 1984.

[4] LeBlanc, L., Mayer, L. Rufino, M. Schock, S., and King, J. Marine sediment classification usign the chirp sonar. J. Acoust. Soc. Am. 91(1), January 1992

[5] Russell, G.T. and Dunbar, R.M. Intelligent control and communication systems for autonomous underwater vehicles. In Proc. 1st Workshop on "Mobile Robots for Subsea Environments". Held at Monterey bay Aquarium, Monterey, CA, USA Oct 23-26 1990.

[6] Steer, B. Steps towards unmanned underwater sensor based robot systems for the exploration and use of the world's oceans. Joint USA-French Workshop on Underwater Robots held in Toulon, France, May 1992.

[7] Steer, B. (with L.R. Leblanc, S.G. Schock) Chirp sonar marine sediment classification sytem for autonomous vehicles. In: Submarine Technology. May 1991. 